\title{
A LOCATION-INVENTORY MODEL INCLUDING DELIVERY DELAY COST AND CAPACITY CONSTRAINTS IN A STOCHASTIC DISTRIBUTION NETWORK
}

\author{
A. Ahmadi Javid ${ }^{1 *}$ and N. Azad ${ }^{2}$ \\ 1,2 Department of Industrial Engineering \\ Amirkabir University of Technology, Iran \\ ahmadi_javid@aut.ac.ir
}

\begin{abstract}
In this paper, we present a distribution network design problem in a supply chain system that minimises the total cost of location, inventory, and delivery delay. Customers' demands are random, and multiple capacity levels are available for the distribution centers. The problem is first formulated as a mixed integer convex programming model to optimally solve medium-sized instances, and then a heuristic is developed for solving large-sized instances.
\end{abstract}

\section{OPSOMMING}

In hierdie artikel word ' $\mathrm{n}$ distribusienetwerkprobleem in ' $\mathrm{n}$ voorsieningsketting voorgehou waar die totale koste van die ligging, voorraad en afleweringsvertragings geminimiseer word. Die vraag is lukraak en verskeie kapasiteitsvlakke is beskikbaar in die verspreidingsentra. Die problem word eers geformuleer as ' $n$ gemengde-heeltal-konvekse model sodat mediumgrootte gevalle geoptimiseer kan word, waarna ' $\mathrm{n}$ heuristieke benadering ontwikkel word vir die oplos van grootskaalse aktiwiteite.

${ }^{*}$ Corresponding author 


\section{INTRODUCTION}

In the past two decades strategic supply chain design and redesign have become a major challenge for firms. Three major cost factors associated with designing and managing a supply chain relate to facility location, inventory control, and distribution. These three cost elements are highly related, and should ideally be considered jointly when making supply chain design decisions. (For an introduction to and review of various types of integration, we refer to Shen [10] and AhmadiJavid and Azad [1].)

Location-inventory integration problems have been studied with interest recently. In these problems, inventory control decisions are incorporated into the facility location decisions. Erlebacher and Meller [4] formulate a non-linear integer location-inventory model. They use a continuous approximation to solve the problem. Teo et al. [15] present an approximation algorithm for the problem of choosing distribution centers to minimise the total cost of location and inventory, ignoring transportation cost. Daskin et al. [3] apply Lagrangian relaxation to solve a location-inventory model. Shen et al. [11] present a location-inventory model that is similar to the model of Daskin et al. [3] and use column generation to solve the problem. Miranda and Garrido [5] present a capacitated location-inventory model that is similar to the model of Daskin et al. [3], and they apply a Lagrangian relaxation method to solve it. Miranda and Garrido [6] extend their earlier location-inventory model (Miranda and Garrido [5]) by introducing more realistic capacity constraints, and solve it by a Lagrangian method. Ozsen et al. [7] also present a capacitated location-inventory model with similar capacity constraints, and solve it by a Lagrangian method. Shu et al. [14] study a more general location-inventory model, and use column generation to solve the problem. Shen [8] proposes a multi-commodity location-inventory model, and solves it by a column generation method. Shen [9] considers a location-inventory with profit maximisation objective, and solves it by a column generation method. Snyder et al. [13] consider a new location-inventory model with risk pooling. Shen and Qi [12] present a location-inventory model whose objective also includes the routing cost, which is approximated, based only on the locations of the opened distribution centers. Recently Ahmadi-Javid and Azad [1] have developed a novel location-inventory-routing model that integrates all the location, inventory, and routing decisions.

In this paper we present an integrated model that simultaneously minimises location, inventory, and delivery delay costs in a supply chain. The delivery delay cost is included in the total cost to take customer responsiveness into account in the design of the network (for another approach to incorporating customer responsiveness along with location, see Ahmadi-Javid and Davoudpour [2].) In fact, the company has to incur some costs if there is a delay in delivering the customers' orders. Moreover, we consider multiple capacity levels for each distribution center to make the problem more realistic. We have shown that the problem, with each type of capacity constraint proposed by Miranda and Garrido ([5] and [6]), can be reformulated as a mixed integer convex program. Finally, we present a hybrid heuristic based on Simulated Annealing and Tabu Search.

The remainder of this paper is organised as follows. In Section 2, the problem is described and formulated. In Section 3, we present the exact and heuristic solution method. Numerical results are given in Section 4. Section 5 concludes the paper.

\section{MODEL FORMULATION}

In this section, we describe and formulate the problem. In Section 2.1 we describe the problem, and in Section 2.2 we present the formulation.

\subsection{Problem description}

Consider a multi-echelon supply chain distribution system composed of a single supplier, multiple distribution centers, and multiple customers. Each customer has an uncertain demand that follows a normal distribution. The goal of our model is to choose a set of opened distribution centers to serve the customers, to allocate the customers to the opened distribution centers, and to determine the inventory policy of each opened distribution center in order to minimise the total 
cost of location, inventory, and delivery delay. The company is liable for delay costs if it delays in meeting the customers' demands. Multiple capacity levels are available for each distribution center. More precisely, we make the following assumptions:

- Each customer has an uncertain demand that follows a normal distribution, and customers' demands are independent of each other.

- The capacity levels for each distribution center are known, and the company pays a fixed location cost for opening a distribution center with a capacity level.

- The distribution centers are assumed to follow a $(Q, R)$ inventory policy, i.e. when the inventory level at a distribution center falls to or below a re-order point $R$, a fixed quantity $Q$ is ordered to the supplier. Also, each distribution center holds a safety stock to buffer the system against stock out during lead times.

- The company pays a fixed cost for placing an order, and holding costs for working inventory and safety stock at each distribution center.

- The company pays a delivery delay cost if it cannot deliver the customer's order within a predefined period of time. The delivery delay cost is linearly dependent on yearly delayed demands.

\subsection{Formulation}

Before presenting the model, let us introduce the notation that will be used throughout this paper.

\subsubsection{Index sets}

$K$ : Set of customers

$J$ : Set of (potential) distribution centers

$N$ : Set of capacity levels available to (potential) distribution centers

\subsubsection{Parameters}

$\mu_{k}:$ Mean of yearly demand at customer $k,(\forall k \in K)$

$\sigma_{k}^{2}$ : Variance of yearly demand at customer $k,(\forall k \in K)$

$f_{j}^{n}$ : Yearly cost for opening and operating distribution center $j$ with capacity level $n$, $(\forall j \in J, \forall n \in N)$

$b_{j}^{n}$ : Capacity with level $n$ for the potential distribution center $j,(\forall j \in J, \forall n \in N)$

$h_{j}$ : Inventory holding cost per unit of product per year at distribution center $j,(\forall j \in J)$

$u_{k}$ : The time within which the goods must be delivered to customer $k$ in years, $(\forall k \in K)$

$t_{j k}$ : The required time for delivering the goods from distribution center $j$ to customer $k$ in years, $(\forall j \in J, \forall k \in K)$

$d_{j k}$ : The indicator showing whether distribution center $j$ delays in serving customer $k$ or not,

$$
\text { i.e. } d_{j k}=\left\{\begin{array}{ll}
1 & \text { if } t_{j k}>u_{k} \\
0 & \text { otherwise }
\end{array} \quad(\forall k \in K, \forall j \in J),(\forall j \in J, \forall k \in K)\right.
$$

$s$ : Penalty cost for delay in delivery of one unit of product per year.

$p_{j}$ : Fixed cost per each order placed with the supplier by distribution center $j,(\forall j \in J)$

$l_{j}$ : Lead time of distribution center $j$ in years, $(\forall j \in J)$

$g_{j}$ : Fixed cost per each shipment from the supplier to distribution center $j,(\forall j \in J)$

$a_{j}$ : Cost for shipment of a unit from the supplier to distribution center $j,(\forall j \in J)$

$c_{j k}$ : Cost for shipment of a unit from distribution center $j$ to customer $k,(\forall j \in J, \forall k \in K)$ 
$\alpha$ : Fill rate considered for inventory systems, $\alpha>0.5$

$\mathbf{z}_{\alpha}$ : Left $\alpha$-percentile of standard normal random variable $Z$, i.e. $\operatorname{Pr}\left(Z \leq \mathbf{z}_{\alpha}\right)=\alpha$

\subsubsection{Decision variables}

$Y_{j k}=\left\{\begin{array}{cc}1 & \text { if customer } k \text { is assigned to distributioncenter } j \\ 0 & \text { otherwise }\end{array}(\forall k \in K, \forall j \in J)\right.$

$U_{j}^{n}=\left\{\begin{array}{lc}1 & \text { if distribution center } j \text { is opened with capacity level } n \\ 0 & \text { otherwise }\end{array}(\forall j \in J, \forall n \in N)\right.$

$Q_{j}$ : Order size at distribution center $j,(\forall j \in J)$

\subsubsection{Elements of objective function}

The objective function minimises the sum of the following costs:

1. The yearly cost of locating distribution centers with capacity level, given by the term $\sum_{j \in J} \sum_{n \in N} f_{j}^{n} U_{j}^{n}$.

2. The shipment cost from distribution centers to customers, given by the term $\sum_{j \in J} \sum_{k \in K} c_{j k} \mu_{k} Y_{j k}$.

3. The expected inventory cost which is the sum of expected working inventory cost and safety stock cost. The expected working inventory cost includes the fixed costs of placing orders and the holding cost of working inventory. Let $D_{j}$ denote the expected total annual demand going through distribution center $j$, i.e. $D_{j}=\sum_{k} \mu_{k} Y_{j k}$. Then the total annual cost of ordering inventory from the supplier to distribution center $j$ is given by $p_{j} \frac{D_{j}}{Q_{j}}+h_{j} \frac{Q_{j}}{2}$. The yearly safety stock cost at distribution center $j$ is given by $h_{j} z_{\alpha} \sqrt{l_{j} \sum_{k} \sigma_{k}^{2} Y_{j k}}$.

4. The expected cost of shipping orders of size $Q$ from the supplier to distribution center $j$ is given by the term $\left(g_{j}+a_{j} Q_{j}\right) \frac{D_{j}}{Q_{j}}$.

5. The expected delivery delay cost is computed as $s \sum_{j \in J} \sum_{k \in K} \mu_{k} Y_{j k}\left(t_{j k}-u_{k}\right) d_{j k}$.

\subsubsection{Mathematical model}

By considering the problem description presented in the previous subsection, the problem can be formulated as follows:

$$
\begin{aligned}
& \min \sum_{j \in J} \sum_{n \in N} f_{j}^{n} U_{j}^{n}+\sum_{j \in J} \sum_{k \in K} c_{j k} \mu_{k} Y_{j k}+\sum_{j \in J}\left[\left(p_{j}+g_{j}\right) \frac{\sum_{k \in K} \mu_{k} Y_{j k}}{Q_{j}}+a_{j} \sum_{k \in K} \mu_{k} Y_{j k}+\frac{h_{j} Q_{j}}{2}+h_{j} z_{\alpha} \sqrt{l_{j} \sum_{k \in K} \sigma_{k}^{2} Y_{j k}}\right] \\
& \quad+s \sum_{j \in J} \sum_{k \in K} \mu_{k} Y_{j k}\left(t_{j k}-u_{k}\right) d_{j k}
\end{aligned}
$$


Subject to:

$$
\begin{array}{lc}
\sum_{j \in J} Y_{j k}=1 & \forall k \in K \\
\sum_{n \in N} U_{j}^{n} \leq 1 & \forall j \in J \\
\sum_{k \in K} \mu_{k} Y_{j k} \leq \sum_{n \in N} b_{j}^{n} U_{j}^{n} \quad \forall j \in J & \forall j \in J, \forall k \in K \\
Y_{j k} \in\{0,1\} & \forall j \in J, \forall n \in N \\
U_{j}^{n} \in\{0,1\} & \forall j \in J \\
Q_{j}>0 &
\end{array}
$$

The model minimises the total expected cost discussed in Section 2.2.4. Constraints (2) guarantee that each customer is assigned to just one distribution center. Constraints (3) ensure that each distribution center can be assigned at most to one capacity level. Constraints (4) ensure that the total expected demand assigned to each opened distribution center does not exceed the capacity assigned to each distribution center. Constraints (5) enforce the integrality restrictions on the binary variables. Constraints (6) enforce non-negativity restrictions on the real valued variables.

We can replace Constraints (4) by the capacity constraints

$$
Q_{j}+z_{\alpha} \sqrt{l_{j} \sum_{k \in K} \sigma_{k}^{2} Y_{j k}} \leq \sum_{n \in N} b_{j}^{n} U_{j}^{n} \quad \forall j \in J
$$

which are more realistic. These constraints ensure that in the worst case the sum of the order size and safety stock of an opened distribution center does not exceed its capacity.

\section{SOLUTION METHODS}

In this section we propose optimal and heuristic solution methods for solving the problem. The optimal solution method is based on convex reformulation of the proposed model in Section 2 as a mixed integer convex program. The heuristic method is a hybridisation based on Simulated Annealing with Tabu Search to solve the large-sized instances of the problem.

\subsection{Optimal solution method}

The model (1)-(6) presented in the previous section is not convex. By observing that the optimal value of $Q_{j}$ is:

$$
Q_{j}^{*}=\sqrt{\frac{2\left(p_{j}+g_{j}\right) \sum_{k \in K} \mu_{k} Y_{j k}}{\theta h_{j}}},
$$

the model (1)-(6) can be rewritten as the following convex program:

$$
\begin{aligned}
\min & \sum_{j \in J} \sum_{n \in N} f_{j}^{n} U_{j}^{n}+\sum_{j \in J} \sum_{k \in K} c_{j k} \mu_{k} Y_{j k}+\sum_{j \in J}\left[\sqrt{2 h_{j}\left(p_{j}+g_{j}\right) \sum_{k \in K} \mu_{k} Y_{j k}^{2}}+a_{j} \sum_{k \in K} \mu_{k} Y_{j k}+h_{j} z_{\alpha} \sqrt{l_{j} \sum_{k \in K} \sigma_{k}^{2} Y_{j k}^{2}}\right] \\
& +s \sum_{j \in J} \sum_{k \in K} \mu_{k} Y_{j k}\left(t_{j k}-u_{k}\right) d_{j k}
\end{aligned}
$$

subject to (2)-(5). 
If we consider Constraints (7) instead of Constraints (4), by noting that

$$
Q_{j}^{*}=\min \left\{\sqrt{\frac{2\left(p_{j}+g_{j}\right) \sum_{k \in K} \mu_{k} Y_{j k}}{\theta h_{j}}}, \sum_{n \in N} b_{j}^{n} U_{j}^{n}-z_{\alpha} \sqrt{l_{j} \sum_{k \in K} \sigma_{k}^{2} Y_{j k}}\right\},
$$

the variables $Q_{j}$ can be eliminated from objective function (1), and then the resulting model can be reformulated as the convex mixed integer program presented below by adding some auxiliary variables and constraints:

$$
\begin{aligned}
\min & \sum_{j \in J} \sum_{n \in N} F_{j}^{n} U_{j}^{n}+\sum_{j \in J} \sum_{k \in K} c_{j k} \mu_{k} Y_{j k}+\sum_{j \in J} \sum_{k \in K} S \mu_{k} Y_{j k}\left(t_{j k}-u_{k}\right) d_{j k} \\
& +\sum_{j \in J}\left[\left(p_{j}+g_{j}\right) W_{j}+a_{j} \sum_{k \in K} \mu_{k} Y_{j k}+\frac{h_{j}}{2}\left(\sqrt{\frac{2\left(p_{j}+g_{j}\right) \sum_{k \in K} \mu_{k} Y_{j k}^{2}}{h_{j}}}-Z_{j}^{-}\right)+h_{j} z_{\alpha} \sqrt{l_{j} \sum_{k \in K} \sigma_{k}^{2} Y_{j k}^{2}}\right]
\end{aligned}
$$

subject to (2), (3), (5) and

$$
\sqrt{\frac{h_{j} \sum_{k \in K} \mu_{k} Y_{j k}^{2}}{2\left(p_{j}+g_{j}\right)}} \leq W_{j} \quad \forall j \in J
$$

$\sum_{k \in K} \mu_{k} \frac{Y_{j k}^{2}}{W_{j}}+z_{\alpha} \sqrt{l_{j} \sum_{k \in K} \sigma_{k}^{2} Y_{j k}^{2}} \leq \sum_{n \in N} b_{j}^{n} U_{j}^{n} \quad \forall j \in J$

$\sum_{n \in N} b_{j}^{n} U_{j}^{n}-z_{\alpha} \sqrt{l_{j} \sum_{k \in K} \sigma_{k}^{2} Y_{j k}}-\sqrt{\frac{2\left(p_{j}+g_{j}\right) \sum_{k \in K} \mu_{k} Y_{j k}}{h_{j}}} \leq Z_{j}^{+}-Z_{j}^{-} \quad \forall j \in J$

$Z_{j}^{-} \leq m V_{j} \quad \forall j \in J$

$Z_{j}^{+} \leq m\left(1-V_{j}\right) \quad \forall j \in J$

$Z_{j}^{+}, Z_{j}^{-} \geq 0 \quad \forall j \in J$

$W_{j}>0 \quad \forall j \in J$

$V_{j} \in\{0,1\} \quad \forall j \in J$

The constant $m$ appearing in Constraints (15) and (16) is a large positive number. Because the relaxations of the models (9), (2)-(5) and (11), (2), (3), (5), (12)-(17) are convex, these models can be solved optimally by any solver that uses the Branch-and-Bound method to solve mixed integer programs.

\subsection{Hybrid heuristic}

Simulated Annealing (SA) and Tabu Search (TS) are two well-known global search heuristic approaches to solve hard combinatorial problems. Here we develop a hybridised SA-TS heuristic based on both SA and TS. The reason for choosing the hybrid heuristic approach is to avoid falling into local optimum traps (using SA) and to avoid search cycling (using TS). Our computational results show that this hybridisation improves the effectiveness of the heuristic. The parameters, steps, procedure for generating initial solutions, and explanation of the moves used in the heuristic are given in the following subsections. 


\subsubsection{Parameters of SA-TS heuristic}

$T_{0}:$ Initial temperature

$T$ : Current temperature

$D R$ : Decreasing rate of temperature

$F T$ : Freezing temperature

$M N$ : Maximum number of visited solution in each temperature

$X_{0}:$ Initial solution

$X$ : Current solution

$X_{n h}:$ A solution in neighbourhood of $X$

$X_{\text {best }}$ : Best solution

$C(X)$ : Objective function value for $X$

\subsubsection{Steps of SA-TS heuristic}

The steps of the proposed hybrid SA-TS heuristic are as follows:

Step1: Select an initial solution $X_{0}$ (see Section 3.2.3), and set $X_{\text {best }}=X_{0}, X=X_{0}$.

Step2: Randomly select a move from Moves $1-4$ (see Section 3.2.4) and by the selected move generate neighbouring solution $X_{n h}$ in the neighbourhood of $X$.

Step3: Is the move selected in Step 2 in the Tabu list? If yes, go to Step 4; otherwise, go to Step 5. Step4: If $C\left(X_{n h}\right) \leq C\left(X_{\text {best }}\right)$ then $X=X_{n h}, X_{\text {best }}=X_{n h}$, update the Tabu list and go to Step 6; otherwise, go to Step 2 to choose another candidate move.

Step5: Set $\Delta C=C\left(X_{n h}\right)-C(X)$.

- 5.1. If $\Delta C \leq 0$, then $X=X_{n h}$, and update the Tabu list. If $C\left(X_{n h}\right)<C\left(X_{\text {best }}\right), X_{\text {best }}=X_{n h}$.

- 5.2. If $\Delta C>0, y \leftarrow U(0,1), z=e^{-\frac{\Delta C}{T}}$. If $y<z$, then $X=X_{n h}$.

Step6: Does the number of the visited neighbouring solution under temperature $T$ exceed $M N$ ? If yes, go to Step 7; if not go to Step 2.

Step7: $T=D R \times T$.

Step8: Is the stopping criterion ( $T<F T$ ) matched? If yes, stop; otherwise, go to Step 2.

\subsubsection{Constructing initial solutions}

To obtain an initial solution, we apply the following steps:

Step 1: Put all the customers into the set $K^{\prime}$.

Step 2: Select randomly a customer from $K^{\prime}$ and delete that customer from $K^{\prime}$.

Step 3: Select a distribution center randomly. If the distribution center is selected for the first time, then select a capacity level for this distribution center randomly.

Step 4: If the remaining capacity of the distribution center selected in Step 3 is greater than the demand of the customer selected in Step 2, then assign the customer to the distribution center and go to Step 5; otherwise, go to Step 3 to select another distribution center.

Step 5: Is $K^{\prime}$ empty? If yes, stop; otherwise, go to Step 2. 


\subsubsection{Moves used to generate neighbouring solutions}

In Step 2 of the proposed SA-TS heuristic we apply four different moves to generate neighbouring solution $X_{n h}$ in the neighbourhood of the current solution $X$. These moves are explained below.

Move 1: Randomly close one of the opened distribution centers and randomly reallocate all of its customers to the remaining opened distribution centers. If the remaining capacities of the other opened distribution centers are not enough to serve the customers of the closed distribution center, then randomly select an opened distribution center and raise its capacity level in order to serve all the customers.

Move 2: Randomly select two opened distribution centers, and exchange their customers. In this move the capacities should be adjusted to serve the new customers.

Move 3: Randomly close one of the opened distribution centers, and assign its customers to a new distribution center that is randomly opened with enough capacity level to serve the assigned customers.

Move 4: Randomly select two opened distribution centers. Then, randomly select one customer from each distribution center and assign it to the other distribution center. In this move the capacities should be adjusted to serve the new customers.

\section{COMPUTATIONAL RESULTS}

In this section we conduct a computational study to assess the performance of the proposed SA-TS heuristic. The test instances are constructed as follows. The means of yearly demands are drawn from a uniform distribution between 250 and 1,250, and the variances are drawn from a uniform distribution between 20 and 200. Also we set the following parameter values:

$h_{j}$ is uniformly drawn from [2, 4],

$p_{j}$ is uniformly drawn from $[15,20]$,

$l_{j}$ is uniformly drawn from [6/365, 10/365],

$g_{j}$ is uniformly drawn from $[15,20]$,

$a_{j}$ is uniformly drawn from [2,5],

$c_{j k}$ is uniformly drawn from $[2,5]$,

$u_{k}$ Is uniformly drawn from $[1,6]$,

$t_{j k}$ is uniformly drawn from $[1,15]$,

$s=2, z_{\alpha}=1.96$ (fill rate is $97.5 \%$ ).

For each potential distribution center four capacity levels are considered. Let $D$ represent the total average of the customers' demands and $|J|$ be the number of the potential distribution centers; then the four capacities for the distribution center $j$ are defined as:

$b_{j}^{1}=\operatorname{cap}(j), b_{j}^{2}=1.5 \times c a p(j), b_{j}^{3}=2 \times c a p(j), b_{j}^{4}=2.5 \times c a p(j)$

where $\operatorname{cap}(j)=\left[c_{j} D /|J|\right]$ and $c_{j}$ is a random number between 0.8 and 1.2 .

The corresponding four fixed set up costs of locating and operating are as follows:

$f_{j}^{1}=\left[0.65 \times k_{j}^{\prime}\right], f_{j}^{2}=\left[0.9 \times k_{j}^{\prime}\right], f_{j}^{3}=\left[1.1 \times k_{j}^{\prime}\right], f_{j}^{4}=\left[1.35 \times k_{j}^{\prime}\right]$ 
where $k_{j}^{\prime}$ is drawn from a uniform distribution between 4,500 and 5,500.

The program of the heuristic method is coded in Visual Basic 6 and run on a Pentium 4 processor with $2.8 \mathrm{~GB}$ RAM. For each instance, we run the heuristic method 20 times; the average objective value is reported in Tables 1 and 2. In the tables 'DC' is the abbreviation for Distribution Center, and the CPU times are in seconds. For each instance the tuning of the parameters of the hybrid heuristic is done by carrying out random experiments.

In Section 4.1 we compare the hybrid SA-TS heuristic with the optimal solution method, and in Section 4.2 we compare the hybrid heuristic with two heuristics based on only SA or TS.

\subsection{Comparison of optimal and heuristic solution methods}

In this section we compare solutions of the proposed heuristic with the optimal solutions obtained by solving model (9), (2)-(5). The model is solved by Lingo 8 . Seventeen instances are solved; the results are given in Table 1 . It can be seen that the solutions of the heuristic are optimal or near optimal in several instances. The average CPU times of the heuristic are considerably less than those of the optimal solution method.

\begin{tabular}{|c|c|c|c|c|c|c|c|}
\hline \multirow[b]{2}{*}{$\#$} & \multirow[b]{2}{*}{ \# Customers } & \multirow[b]{2}{*}{ \# Potential DCs } & \multicolumn{2}{|c|}{ Optimal method } & \multicolumn{3}{|c|}{ Heuristic method } \\
\hline & & & Cost & CPU time & Cost & CPU time & Error $(\%)$ \\
\hline 1 & 4 & 2 & 19754.3 & 3 & 19754.3 & 1 & 0.00 \\
\hline 2 & 6 & 3 & 24322.6 & 7 & 24322.6 & 3 & 0.00 \\
\hline 3 & 7 & 3 & 27462.7 & 13 & 27462.7 & 5 & 0.00 \\
\hline 4 & 8 & 4 & 32841.5 & 18 & 32841.5 & 9 & 0.00 \\
\hline 5 & 9 & 4 & 36528.4 & 39 & 36528.4 & 11 & 0.00 \\
\hline 6 & 20 & 5 & 73938.6 & 121 & 73938.6 & 26 & 0.00 \\
\hline 7 & 30 & 8 & 121463.1 & 183 & 122279.8 & 35 & 0.67 \\
\hline 8 & 40 & 12 & 176413.6 & 297 & 177692.5 & 47 & 0.72 \\
\hline 9 & 50 & 15 & 218175.2 & 429 & 220416.7 & 61 & 1.03 \\
\hline 10 & 60 & 17 & 269745.2 & 981 & 272456.3 & 74 & 1.01 \\
\hline 11 & 70 & 19 & 303951.5 & 1936 & 307424.8 & 89 & 1.14 \\
\hline 12 & 80 & 21 & 362431.6 & 2973 & 366826.1 & 104 & 1.21 \\
\hline 13 & 90 & 23 & 406682.4 & 5103 & 411894.3 & 120 & 1.28 \\
\hline 14 & 100 & 25 & 471295.3 & 2 hours limit & 450223.7 & 137 & - \\
\hline 15 & 120 & 30 & 577384.5 & 2 hours limit & 534793.2 & 171 & - \\
\hline 16 & 150 & 35 & 751270.2 & 2 hours limit & 672364.1 & 221 & - \\
\hline 17 & 180 & 38 & 897841.3 & 2 hours limit & 792199.5 & 272 & - \\
\hline
\end{tabular}

Table 1: Comparison of optimal and hybrid heuristic solution methods

\subsection{Comparison of hybrid heuristic with SA and TS heuristics}

In this section, we compare the hybrid heuristic with a heuristic based on SA or TS. The procedures for obtaining initial solutions and candidate moves in the SA and TS heuristics are similar to the ones exploited in the hybrid SA-TS heuristic. For each instance, the tuning of the parameters of both heuristics is done by carrying out random experiments. In Table 2 we compare the three heuristics. It can be seen that the solution quality of the hybrid heuristic is better than both SA and TS heuristics. Moreover, we can see that the SA heuristic is better than the TS heuristic in both solution quality and CPU time. 


\begin{tabular}{|c|c|c|c|c|c|c|c|c|}
\hline \multirow[b]{2}{*}{ \# } & \multirow[b]{2}{*}{ \# Customers } & \multirow[b]{2}{*}{ \# Potential DCs } & \multicolumn{2}{|c|}{ Hybrid } & \multicolumn{2}{|c|}{ SA } & \multicolumn{2}{|c|}{ TS } \\
\hline & & & Cost & CPU Time & Cost & $\overline{\text { CPU Time }}$ & Cost & CPU Time \\
\hline 1 & 30 & 8 & 122279.8 & 35 & 133763.5 & 34 & 133957.1 & 34 \\
\hline 2 & 40 & 12 & 177692.5 & 47 & 179658.4 & 45 & 179563.2 & 46 \\
\hline 3 & 50 & 15 & 220416.7 & 61 & 223731.5 & 58 & 224043.6 & 59 \\
\hline 4 & 60 & 17 & 272456.3 & 74 & 276261.1 & 71 & 277168.9 & 72 \\
\hline 5 & 70 & 19 & 307424.8 & 89 & 313594.8 & 86 & 315816.3 & 87 \\
\hline 6 & 80 & 21 & 366826.1 & 104 & 374543.2 & 100 & 377197.6 & 101 \\
\hline 7 & 90 & 23 & 411894.3 & 120 & 421197.4 & 115 & 423864.8 & 118 \\
\hline 8 & 100 & 25 & 450223.7 & 137 & 461451.8 & 132 & 465376.3 & 136 \\
\hline 9 & 120 & 30 & 534793.2 & 171 & 544817.5 & 164 & 546362.2 & 169 \\
\hline 10 & 150 & 35 & 672364.1 & 221 & 685234.8 & 214 & 687023.9 & 216 \\
\hline 11 & 180 & 38 & 792199.5 & 272 & 809143.6 & 264 & 811215.4 & 269 \\
\hline 12 & 200 & 40 & 853423.1 & 308 & 872816.4 & 300 & 878942.5 & 305 \\
\hline 13 & 230 & 43 & 961957.8 & 361 & 986095.5 & 352 & 995364.1 & 357 \\
\hline 14 & 250 & 45 & 1039612 & 400 & 1068794 & 391 & 1080246 & 398 \\
\hline 15 & 280 & 48 & 1154184 & 454 & 1192313 & 444 & 1213854 & 450 \\
\hline 16 & 300 & 50 & 1235617 & 494 & 1284570 & 482 & 1312826 & 486 \\
\hline
\end{tabular}

Table 2: Comparison of hybrid SA-TS heuristic with SA and TS heuristics

\section{CONCLUSIONS}

In this paper we consider an integrated location-inventory model in a supply chain distribution network. The network is a multi-echelon supply chain distribution system composed of a single supplier, multiple distribution centers, and multiple customers. Multiple capacity levels for each distribution center are available. The goal of the integrated model is to choose a set of opened distribution centers to serve the customers, to allocate the customers to the opened distribution centers, and to determine the inventory policy of each opened distribution center such that the total cost of location, inventory, and delivery delay is minimised. The delivery delay cost is included in the total cost to take customer responsiveness into account during the design of the network. Considering multiple capacity levels for each distribution center makes the problem more realistic.

We have shown that the problem can be modelled as a mixed integer convex program. We also present a hybrid heuristic based on Simulated Annealing and Tabu Search. We study the integer programming model and hybrid heuristic by conducting a numerical experiment. The numerical experiment indicates that the model can be used to solve optimally medium-sized instances; moreover, it shows that the hybrid heuristic is both effective and efficient.

\section{REFERENCES}

[1] Ahmadi Javid,A. \& Azad, N. 2010. Incorporating location, routing and inventory decisions in supply chain network design, Transportation Research, Part E, 46, pp 582-597.

[2] Ahmadi Javid, A. \& Davoudpour, H. 2009. A new model for single facility location based on service level, South African Journal of Industrial Engineering, 20, pp 219-227.

[3] Daskin, M., Coullard, C. \& Shen, Z.J. 2002. An inventory-location model: Formulation, solution algorithm and computational results, Annals of Operations Research, 110, pp 83106.

[4] Erlebacher, S.J. \& Meller, R.D. 2000. The interaction of location and inventory in designing distribution systems, IIE Transactions, 32, pp 155-166.

[5] Miranda, P.A. \& Garrido, R.A. 2004. Incorporating inventory control decisions into a strategic distribution network design model with stochastic demand, Transportation Research, Part E, 40, pp 183-207. 
[6] Miranda, P.A. \& Garrido, R.A. 2008. Valid inequalities for Lagrangian relaxation in an inventory location problem with stochastic capacity, Transportation Research, Part E, 44, pp 47-65.

[7] Ozsen, L., Daskin, M.S. \& Coullard, C.R. 2006. Capacitated warehouse location model with risk pooling, Naval Research Logistics, 55, pp 295-312.

[8] Shen, Z.J. 2005. A multi-commodity supply chain design problem, IIE Transactions, 37, pp 753-762.

[9] Shen, Z.J. 2006. A profit maximizing supply chain network design model with demand choice flexibility, Operations Research Letters, 34, pp 673-682.

[10] Shen, Z.J. 2007. Integrated supply chain design models: A survey and future research directions, Journal Of Industrial And Management Optimization, 3, pp 1-27.

[11] Shen, Z.J., Coullard, C. \& Daskin, M. 2003. A joint location-inventory model, Transportation Science, 37, pp 40-55.

[12] Shen, Z. \& $Q \mathbf{i}$, L. 2007. Incorporating inventory and routing cost in strategic location models, European Journal of Operational Research, 179, pp 372-389.

[13] Snyder, L.V., Daskin, M.S. \& Teo, C.P. 2007. The stochastic location model with risk pooling, European Journal of Operational Research, 179, pp 1221-1238.

[14] Shu, J., Teo, C.P. \& Shen, Z.J. 2005. Stochastic transportation-inventory network design problem, Operations Research 53, pp 48-60.

[15] Teo, C.P., Ou, J. \& Goh, M. 2001. Impact on inventory costs with consolidation of distribution centers, IIE Transactions 33, pp 99-110. 
http://sajie.journals.ac.za 